\title{
INTERAÇÃO DO HOMEM COM A MANUFATURA POR INTERMÉDIO DE PROTÓTIPOS
}

\author{
Leonardo Macarrão Junior ${ }^{1}$, Paulo Carlos Kaminski ${ }^{2}$ \\ ${ }^{1}$ ESEG - Escola Superior de Engenharia e Gestão \\ ${ }^{2}$ Universidade de São Paulo - Escola Politécnica
}

E-mails: leonardo.macarrao@eseg.edu.br; pckamins@poli.usp.br

\begin{abstract}
RESUMO
Os protótipos são fundamentais ao desenvolvimento do produto e do seu processo de fabricação, por permitirem a melhor interação entre o projetista com a sua criação, de forma a melhor atender ao objetivo final para o qual um determinado componente ou produto é concebido. Também têm fundamental importância por encurtar etapas do desenvolvimento, ao proporcionar um melhor entendimento das questões de projeto, facilitando não só o desenvolvimento do produto propriamente dito, como também identificar eventuais problemas aos quais estão sujeitos o produto ou o processo de fabricação. Ao identificar estes problemas, é possível trabalhar para evitar que eles aconteçam no futuro, reduzindo assim o prazo de lançamento do produto e, principalmente, seus custos. Neste processo está inserido o homem, que será o responsável pela fabricação seriada de peças e sua montagem durante todo o período que o produto for produzido e comercializado. Nas operações não robotizadas, é fundamental que o processo de fabricação esteja em acordo com as questões ergonômicas e não proporcionem riscos àqueles que fazem parte do processo de manufatura. Este trabalho mostra como os mock-ups que são modelos não funcionais, os protótipos físicos funcionais e os protótipos virtuais podem auxiliar nesta interação do produto e processo com o homem. Propor que a utilização do protótipo seja também para avaliar e definir condições adequadas à ergonomia do operador e de todos os que interagem com o produto no processo produtivo, prever e evitar riscos e proporcionar condições de trabalho seguras e favoráveis. Na grande maioria das vezes, os ganhos desta interação estão relacionados às condições inadequadas que são evitadas e não têm como serem mensurados no curto prazo. Sem dúvida, significam a ausência de surpresas desagradáveis a médio e longo prazo ao processo de manufatura e principalmente ao preservar a integridade física e a saúde do ser humano.
\end{abstract}

\section{INTRODUÇÃO}

Qualquer que seja o produto, fabricado em pequenos ou grandes volumes, sua fabricação precisa ser realizada em um ambiente seguro. De acordo com as leis, as companhias precisam gerenciar os riscos e os perigos do ambiente de trabalho de cada operação fabril, a fim de prover a segurança e proteger a saúde de seus empregados [1].

O desenvolvimento de um produto acompanhado do desenvolvimento do seu processo de fabricação tem sua produção dentro dos padrões estipulados e em um ambiente seguro. Portanto, o desenvolvimento completo estende-se até a fase de validação do produto e do processo de fabricação, prontos para o início da manufatura em série. 
O protótipo é fundamental neste processo. Sua função básica é encurtar etapas do desenvolvimento, entre o início do desenvolvimento do produto até o início da produção seriada [2]. Porém, além disso, o protótipo pode desempenhar função nas avaliações ergonômicas das operações envolvidas, visando à preservação da saúde do operador e evitando qualquer tipo de doenças ocupacionais, como lesão por esforços repetitivos - LER ou outras doenças que possam comprometer a saúde futura do operador.

Neste desenvolvimento, um protótipo pode ser físico ou virtual. Enquanto um protótipo físico permite a interação com o produto, por intermédio do manuseio, um protótipo virtual, por sua vez, permite de maneira similar, a interação com o produto, processo de fabricação e a simulação do ambiente de trabalho por meio da realidade virtual [3].

A aplicação mais significativa da utilização de protótipos está na indústria automotiva, devido aos grandes volumes produzidos e ao alto grau de complexidade de um automóvel. Eventuais problemas de ordem ergonômica, por menores que sejam, são potencializados na produção de grandes volumes. Entretanto, estes conceitos também podem ser aplicados a qualquer tipo de produto que seja fabricado em qualquer volume, seja o produto simples ou complexo e em qualquer segmento do mercado.

\section{PROTÓTIPOS NO DESENVOLVIMENTO DE PRODUTOS}

Embora haja diferentes entendimentos de nomenclatura entre os diversos autores, este artigo adota e considera mock-ups como modelos físicos não funcionais que antecedem a fase de protótipos. Eles têm a finalidade de auxiliar o desenvolvimento do produto em suas fases iniciais. São modelos tridimensionais, sem detalhes construtivos, que oferecem o benefício adicional de análise de forma, tamanho, posicionamento e ajustes com as demais peças que compõem o ambiente onde a peça ou sistema será aplicado [4]. Mesmo que ainda seja apenas um conceito, permitirá ao projetista analisar como será o produto final, conforme exemplo mostrado na figura 1 .
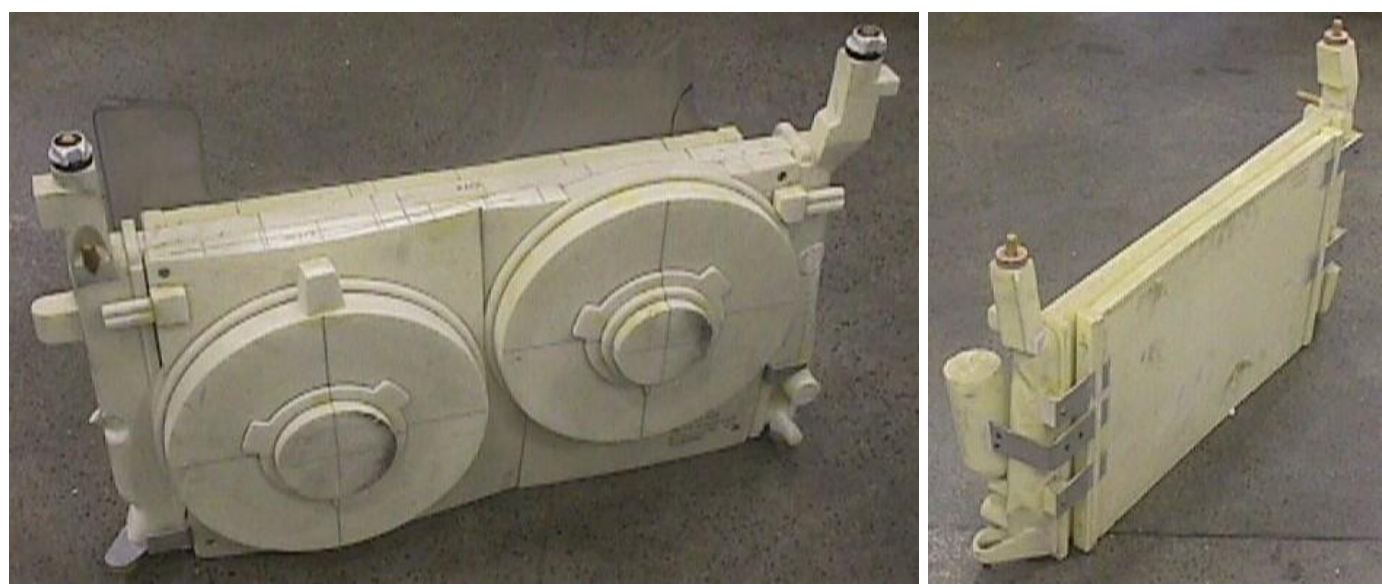

Figura 1 - Vistas do mock-up em poliuretano do conjunto radiador e seus ventiladores.

Já um protótipo propriamente dito deve ser funcional. Geralmente são os primeiros exemplares fabricados individualmente com o propósito de servir de testes [5]. Em casos específicos, um protótipo pode até ser comercializado [6]. As principais finalidades de um protótipo são aprendizagem, comunicação, integração, redução de tempo, de custo e dos riscos da inovação, com o propósito de encurtar as etapas do desenvolvimento do produto [2]. 
São utilizados para testes funcionais e de validação nas fases avançadas do desenvolvimento do produto [7].

Mesmo considerando o custo envolvido de um protótipo físico, os benefícios obtidos, embora nem sempre possam ser quantificados [2], são significativos ao evitar problemas no produto, manufatura e prazo de desenvolvimento. A fim de reduzir estes custos, alguns recursos podem ser utilizados, como por exemplo, a fabricação de um protótipo em escala reduzida. Também pode ser considerada a fabricação um protótipo parcial ou ainda um protótipo capaz de desempenhar apenas as funções que precisam ser testadas [8].

Os protótipos virtuais são criados com o uso de softwares de desenho e simulação eletrônica. Surgiram com o objetivo de reduzir os custos de fabricação de um protótipo físico. São muito úteis se tiverem sua aplicação combinada com os protótipos físicos, pois sua obtenção é mais rápida e tem menor custo, apesar de estarem limitados à disponibilidade dos recursos digitais [9]. Um protótipo virtual também permite verificar cada componente e sua montagem em subsistemas (Figura 2a), identificando precocemente eventuais problemas de montagem como interferências, folgas e outras inconsistências de projeto. Softwares específicos permitem efetuar análises estruturais entre outras avaliações (Figura 2b).

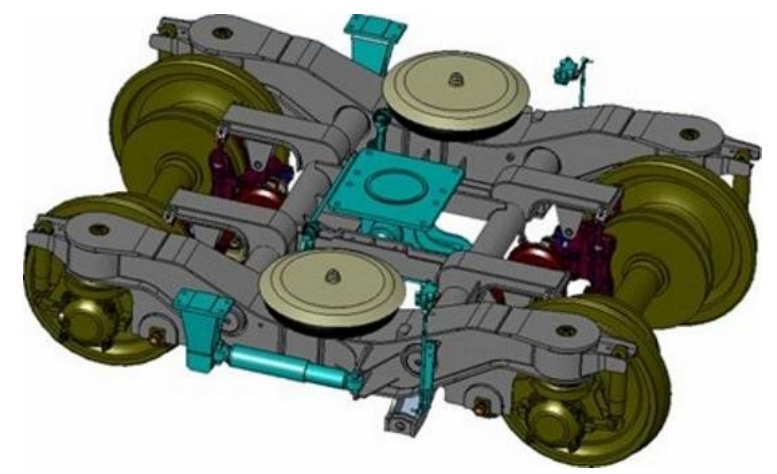

Figura $2 \mathrm{a}$ - Protótipo virtual do conjunto de eixo/roda de trem. [10].

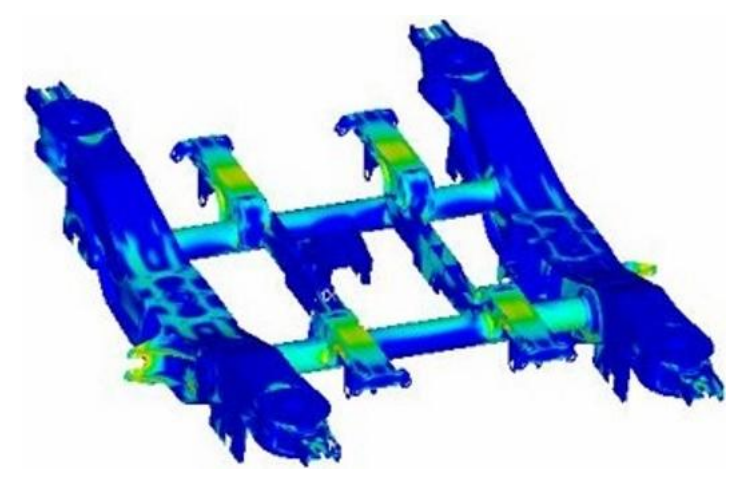

Figura $2 b$ - Análise virtual de fadiga da estrutura do eixo de trem. [10].

Em algumas fases específicas do desenvolvimento do produto, especialmente em produtos mais simples, os protótipos virtuais podem resolver de maneira satisfatória sem a necessidade de protótipos físicos. Em avaliações complexas, fornecem informações para que um protótipo físico seja utilizado apenas na fase subsequente do desenvolvimento. Algumas avaliações somente apresentarão um resultado confiável quando realizadas fisicamente [11].

\section{ERGONOMIA}

De acordo com a Associação Internacional de Ergonomia, "a Ergonomia (ou Fator Humano) é uma disciplina científica relacionada ao entendimento das interações entre os seres humanos e outros elementos ou sistemas e à aplicação de teorias, princípios, dados e métodos a projetos a fim de otimizar o bem estar humano e o desempenho global do sistema". Em especial a ergonomia física, relacionada à anatomia do ser humano e a ergonomia cognitiva, que se referem aos processos mentais, tais como percepção, memória, raciocínio e respostas motoras [12]. 
$\mathrm{Na}$ execução de qualquer atividade por um trabalhador, devem ser considerados os fatores externos, como os objetivos determinados pela empresa e os recursos disponíveis para a execução das tarefas e os fatores internos, como as propriedades gerais do organismo humano, do raciocínio, conhecimentos e experiências e a personalidade [13].

Mesmo considerando estes conceitos e as legislações pertinentes de cada país, na Europa, 47,5\% dos operadores de linha de montagem sofrem com dores musculares ou na parte inferior da coluna vertebral. Por esta razão, deu-se início à proposta de simulação do processo de fabricação com a utilização de modelos humanos [14]. A situação se torna mais crítica à medida que aumenta a idade de aposentadoria dos operadores [15].

\section{DESENVOLVIMENTO DO PROCESSO DE FABRICAÇÃO E MANUFATURA}

Processo de fabricação pode ser definido como uma mudança nas propriedades de um objeto, tais como geometria, estado, informação contida, entre outros, utilizando três agentes essenciais: material, energia e informação. Já um sistema de manufatura pode ser definido como uma série de processos de fabricação com adição de valores transformando matériaprima em produtos acabados [16].

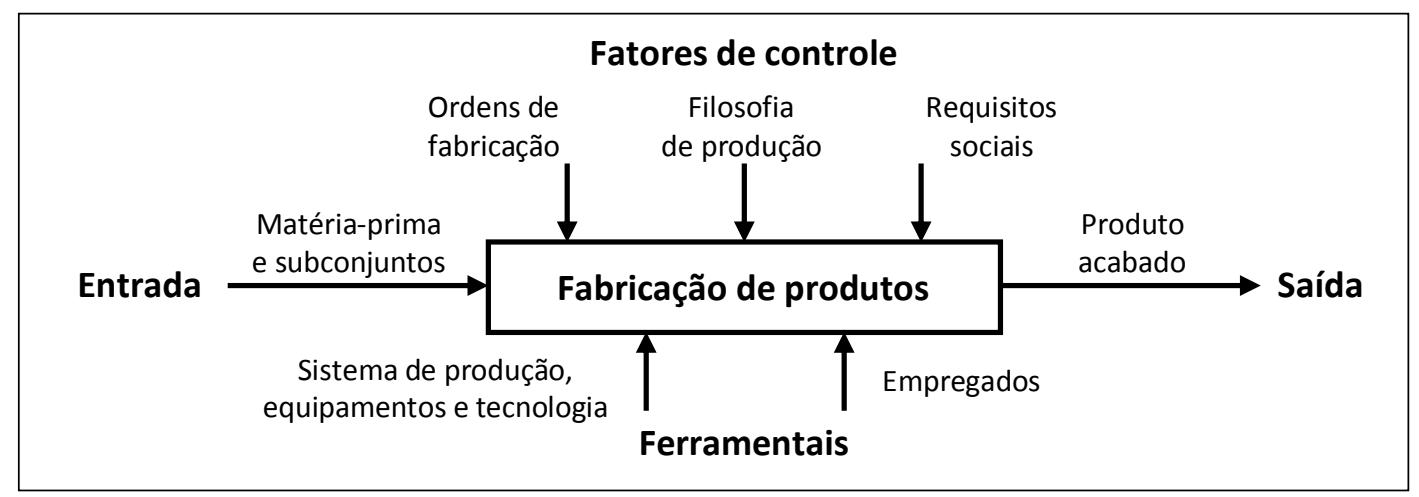

Figura 3 - Modelo de um sistema de manufatura. Adaptado de [16].

A figura 3 mostra que o sistema de manufatura é basicamente controlado por três fatores [16]:

1. Ordens de fabricação determinam o que se espera produzir, de acordo com o mercado.

2. Filosofia de produção aplicada contém o controle e o gerenciamento da manufatura.

3. Requisitos sociais, pressões, proteção ao ambiente e a melhoria das condições de trabalho.

Os fatores citados no item 3 acima são considerados na fase de preparação de máquinas e equipamentos devido ao grande impacto no desenvolvimento do sistema de produção e tecnologia aplicada à manufatura.

Este contexto mostra a relevância das condições humanas no processo produtivo. Considerar o investimento em equipamentos auxiliares que diminuam os esforços físicos e evitem os movimentos mais agressivos dos operadores é tão importante quanto a garantia da qualidade e demais características técnicas do produto. Melhores condições de trabalho e ergonomia do trabalhador tendem a reduzir os afastamentos por problemas de saúde, proporcionar benefícios à produção e ao produto [17], [18], além de evitar problemas judiciais entre empregado e empregador. 
Operações com condições de médio ou alto risco ao trabalhador, assim como as que não sejam favoráveis à ergonomia merecem especial atenção. Nestes casos é indicado maior grau de automação, nos quais, eventualmente, a robotização completa da operação deverá ser considerada. Se o desenvolvimento do processo de fabricação e da manufatura não partir destas premissas, estará sujeito a sérios problemas durante o todo o período de execução das operações fabris.

\section{ANÁLISE DA ERGONOMIA COM APLICAÇÃO DO PROTÓTIPO}

Há algum tempo atrás, o estudo ergonômico restringia-se aos especialistas em ergonomia e somente era aplicado quando a melhor eficiência de produção, utilidade e qualidade já haviam sido alcançadas [3].

Observou-se que os protótipos físicos, construídos originalmente para desenvolvimento de processo de fabricação, poderiam também ser utilizados para avaliações da ergonomia da operação, como por exemplo, o mock-up de uma pinça de solda (Figura 4). Construído papelão em escala natural originalmente para estudo de acesso às peças em chapa metálica a serem soldadas, também permite identificar uma situação de ergonomia inadequada. Nesta fase do desenvolvimento do processo ainda há tempo suficiente para que ações de melhoria sejam tomadas sem que haja interferência na data de início de produção.

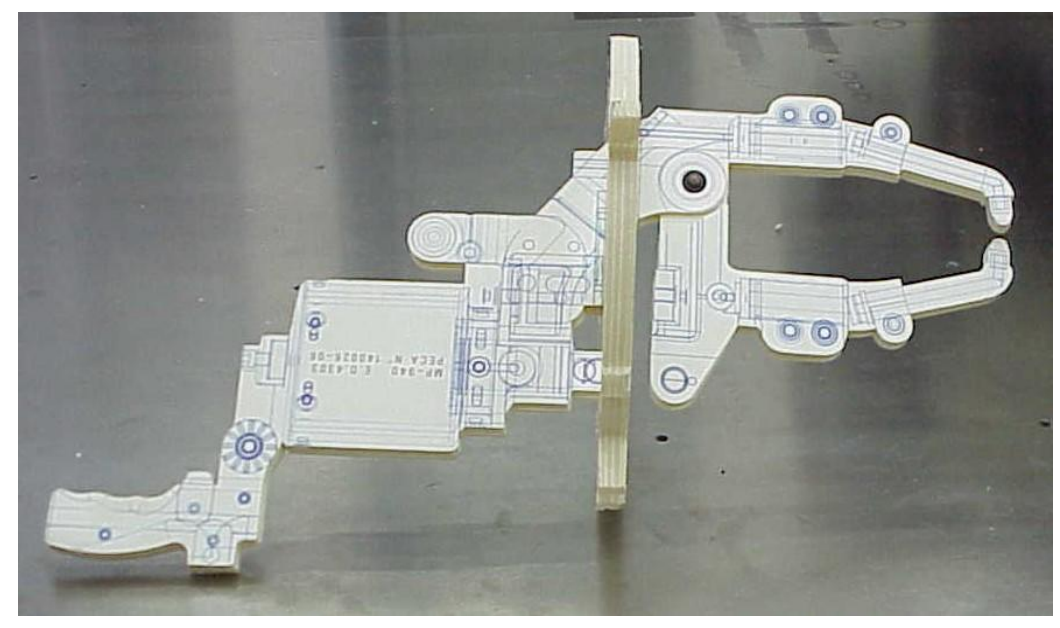

Figura 4 - Mock-up em papelão de uma pinça de solda em escala natural.

Para antecipar cada vez mais eventuais problemas de manufatura, as correções que antes eram efetuadas após a aparição do problema, chamadas de abordagem reativa, deram lugar à abordagem proativa, simplesmente pela possibilidade de prever eventual problema antes que ele realmente seja um problema. Estima-se que $90 \%$ dos problemas podem ser identificados nesta fase, refletindo em significativa redução de custos de instalações [19].

Atualmente os desenvolvimentos contam com a utilização das fábricas digitais, que são softwares utilizados para o desenvolvimento, simulação e validação do processo de manufatura [6]. São aplicados especialmente a produtos com grande volume de produção. Auxiliam no desenvolvimento do processo propriamente dito e simulam as operações, proporcionando a possibilidade de análise das condições ergonômicas dos operadores, como a postura, movimentos, esforços, distâncias percorridas entre outros fatores conforme mostrado nas figuras $5 \mathrm{a}$ e $5 \mathrm{~b}$. 


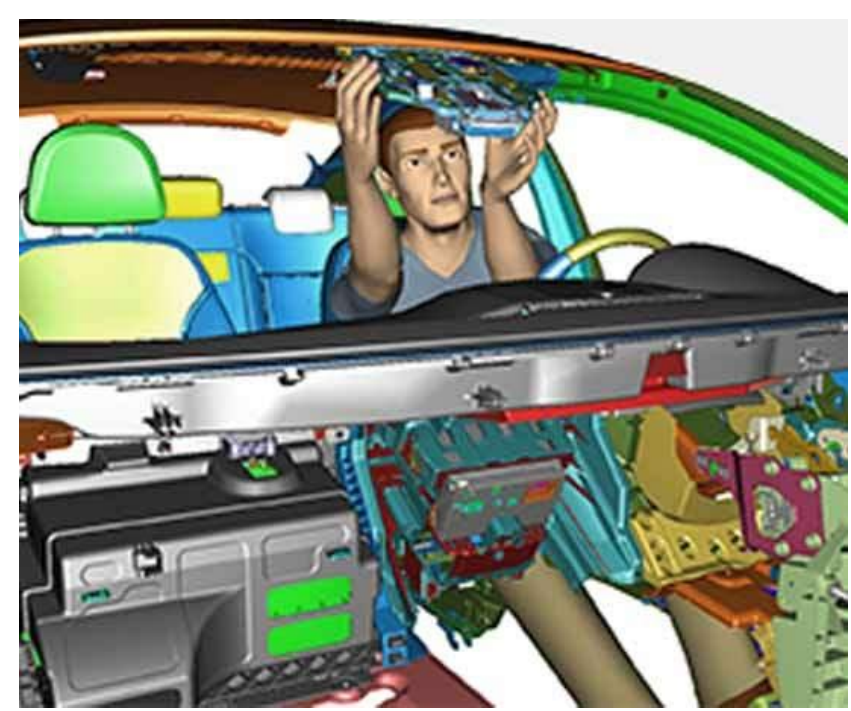

Figura 5a - Simulação de montagem de componentes no teto interno de um veículo [20].

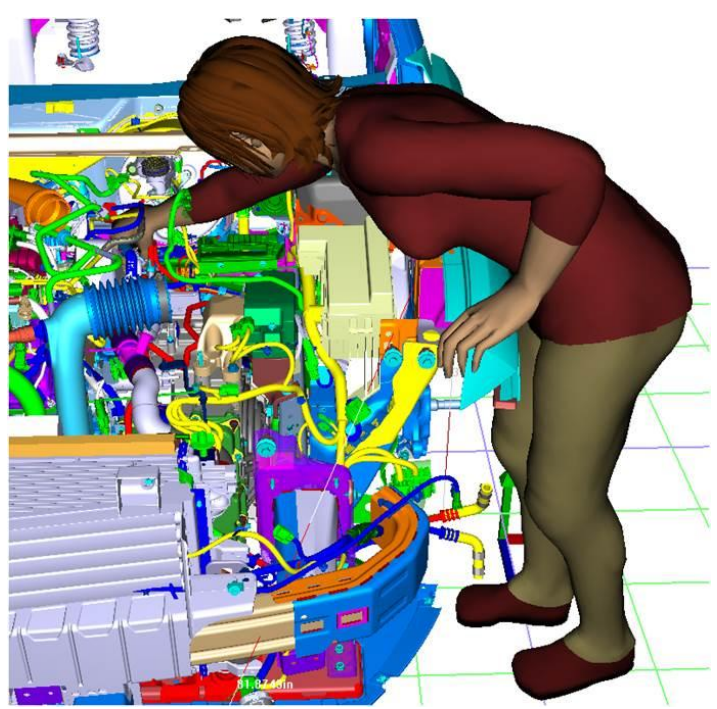

Figura $5 b$ - Simulação de montagem de componentes no compartimento do motor de um veículo [17].

Mesmo com a manufatura já em andamento, é possível conseguir ganhos significativos com esforços adicionais. Como exemplo, com a aplicação da manufatura digital, a Ford conseguiu reduzir em $70 \%$ a taxa de lesões em trabalhadores das duas fábricas em solo norte americano. Esse resultado foi obtido com a introdução de novos dispositivos auxiliares, baseados nos estudos ergonômicos de softwares [17].

Qualquer alteração de um processo de fabricação já em andamento gera custos considerados adicionais. A empresa tem custos para instalar sua linha de produção e, tempos depois, tem novos custos para implementar melhorias nesta mesma linha de produção. Isso pode afetar a qualidade e o processo de fabricação, além da possibilidade de gerar problemas de saúde aos seus funcionários.

A simulação das operações utilizando modelos digitais de humanos permitiu identificar eventuais problemas de postura devido à possibilidade de simular grande variação de movimentos durante a execução futura das operações. Isso permitiu mapear as posturas críticas com potencial risco de doenças nas articulações e musculatura [21]. Ela mostra como um operário irá trabalhar em seu posto de trabalho, os tempos, sequência de trabalho, otimização de ferramentas e dispositivos e relacionada à parte humana, seus movimentos, esforços, fadiga, riscos de acidentes, entre tantas outros fatores [19].

Para auxiliar as verificações gráficas, conforme mostrado nas figuras 5 acima, as análises ergonômicas também podem ser realizadas com a captação virtual dos movimentos utilizando modelos humanos reais (figuras 6 e 7), que oferecem um estudo mais preciso com a captação real dos movimentos para auxílio na análise virtual, por intermédio de sensores de leitura de posição [3], [17].

Estes estudos podem ser realizados ainda antes, na fase inicial dos mock-ups virtuais, oferencendo referências de movimentos ergonômicos para que produto e processo sejam concebidos o mais próximo possível da condição ideal. 


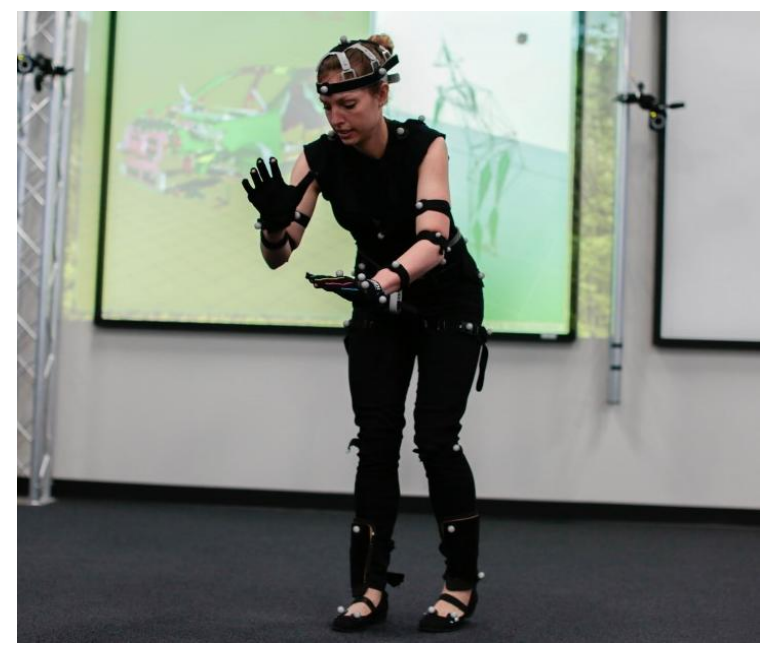

Figura 6 - Captura por sensores da movimentação e amplitude de movimentos de todo o corpo do operador [17].

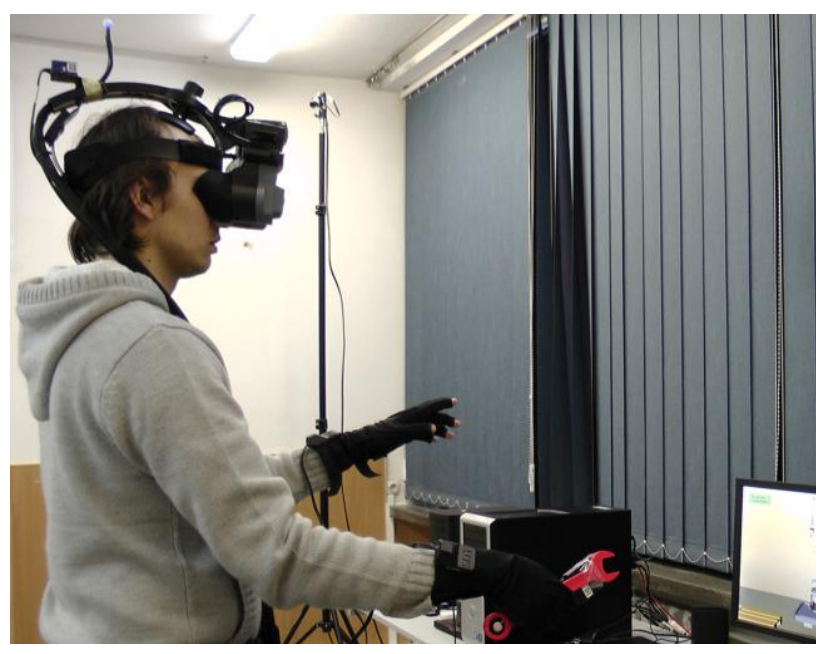

Figura 7 - Captura por sensores de movimento de operação específica, com utilização de óculos de simulação 3D [3].

Há situações em que apenas a captação de movimentos para estudos virtuais não é suficiente. Mesmo com a utilização de óculos de simulação 3D, os movimentos captados podem ter resultado diferente de real. Nestes casos, podem ser combinadas a utilização da prototipagem física e virtual, conforme já citada acima. A figura 8 mostra um mock-up físico, não funcional fabricado em resina de poliuretano, utilizado como auxiliar para captação de movimentos. Isso permite que as simulações de movimentos e esforços seja realizada de maneira muito próxima da real a ser vivenciada pelo operador na linha de produção seriada [9], [17].

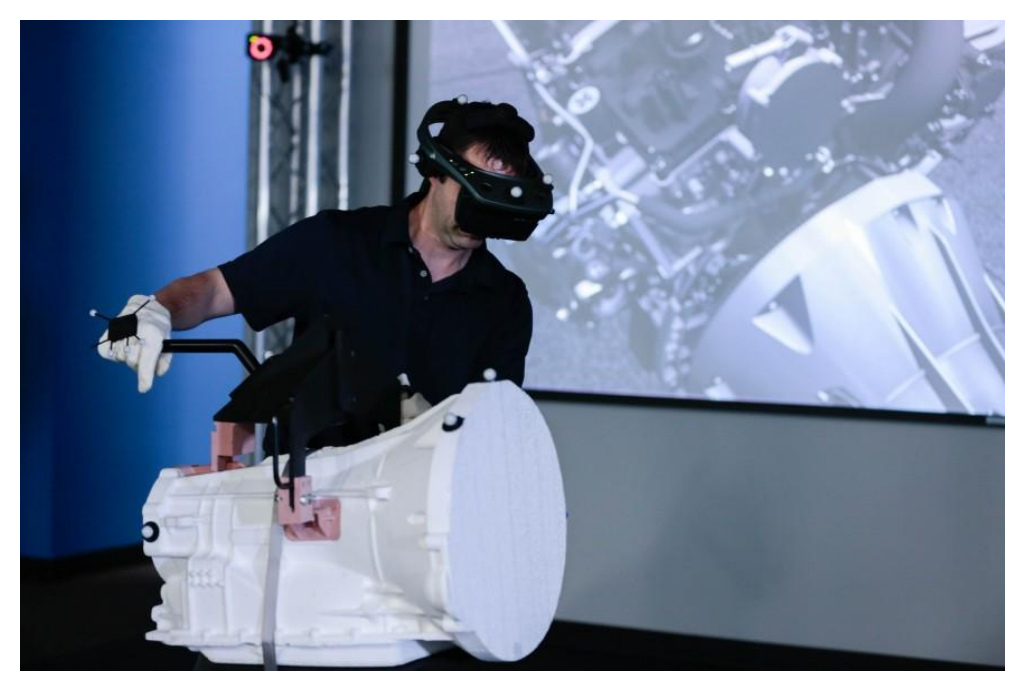

Figura 8 - Captura por sensores de movimento de operação específica, com utilização de óculos de simulação 3D e mock-up não funcional [17].

Os estudos ergonômicos também podem se estender às operações de manutenção. Análises com protótipos físicos e as simulações virtuais sinalizam as condições de acesso de ferramentas para manutenção, espaço reservado, viabilidade e tempo de operação. Da mesma maneira, oferecem o benefício de análise ergonômica do operador que realizará a manutenção. Vale ressaltar que as condições de manutenção geralmente são bastante diferentes das condições de montagem do produto e a manutenção deve contemplar as operações de desmontagem e montagem dos componentes a serem substituídos. 
A análise das condições da manutenção deve ser realizada ainda na fase de desenvolvimento do produto, com a utilização de protótipos físicos ou com as simulações virtuais. A inclusão da análise ergonômica da manutenção garante a segurança, conforto e desempenho do operador [22]. A figura 9 mostra uma simulação de reparo no motor de um veículo, com análise do acesso de ferramenta, da posição da cabeça, braço e esforços do operador.

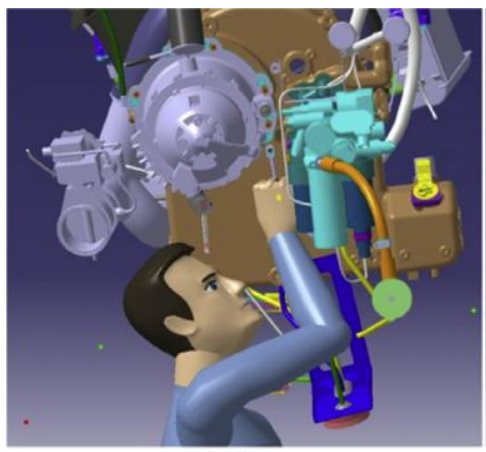

(a)

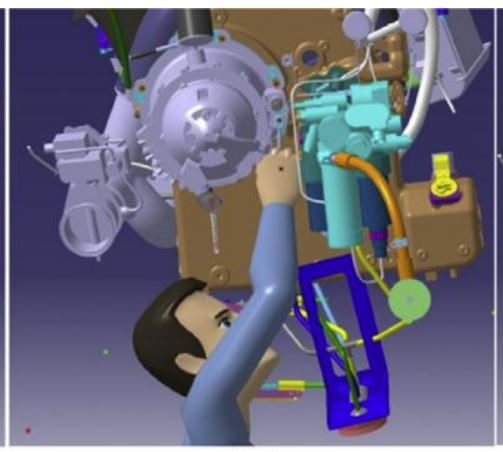

(b)

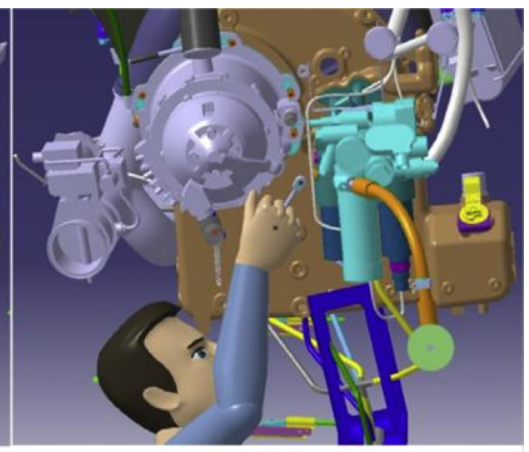

(c)

Figura 9 - Simulação da sequência do processo de manutenção do motor de um veículo [22].

\section{CONCLUSÃO}

A utilização das fases de prototipagem física ou virtual do desenvolvimento do produto no processo de avaliação ergonômica traz benefícios adicionais quando utilizados no desenvolvimento das condições de ergonomia dos operadores. A partir da simulação, conseguem identificar e corrigir situações ergonômicas desfavoráveis e evitar a configuração de condições inseguras.

O aumento no trabalho, no tempo e no custo de simulações e análises de ergonomia de operações durante a fase do desenvolvimento do produto é muito pequeno quando comparado aos ganhos obtidos nas fases de manufatura e manutenção do produto. Os ganhos obtidos nem sempre podem ser mensurados e quantificados adequadamente, pois quando se evita um problema, suas consequências não serão observadas. Mesmo assim não é difícil observar que condições adequadas de ergonomia e segurança do trabalho acarretam em melhores condições de saúde dos operadores, menor absenteísmo por doenças de trabalho e risco de acidentes durante as operações de montagem, manuseio ou manutenção.

O custo das correções e modificações de postos de trabalho por problemas de segurança e ergonomia devem considerar o custo de aquisição e instalação dos equipamentos e ferramentas, somado ao custo de aquisição e instalação de novas benfeitorias, alterações de layout da fábrica. Ainda deve ser considerada a quebra da cadência produtiva para alterações de instalações e layout e o risco de afastamento de operador em decorrência de eventuais acidentes ou lesões musculares. Eventual problema judicial entre empregado e empregador em decorrência de doenças ocupacionais não devem ser descartados.

Os gastos com a utilização de protótipos sejam eles físicos ou virtuais nas fases iniciais do desenvolvimento do produto são justificados porque evitam paradas de linha de montagem para correções, análise de manutenção, modificações de produto e recall e melhoram o ambiente de trabalho. 
Ambiente seguro é mais produtivo. Estimula os operadores a evitarem as atitudes inseguras diminuindo os riscos de acidentes de trabalho. Embora em empresas grandes pequenos problemas sejam potencializados pelos altos volumes de produção, a cultura de segurança não pode depender do faturamento ou tamanho da empesa nem do número de empregados. $\mathrm{O}$ investimento em segurança jamais deve ser reduzido, pois a saúde e a importância do trabalhador independem do local em que ele atue.

\section{REFERÊNCIAS}

[1] NORDLÖF, Hasse; WIITAVAARA, Birgitta; HÖGBERG, Hans; WESTERLING, Ragnar. A cross-sectional study of factors influencing occupational health and safety management practices in companies. Safety Science Journal - Elsevier, V.95, p.92-103, 2017.

[2] VOLPATO, Neri. Prototipagem rápida / ferramental rápido no processo de desenvolvimento de produto. Revista Máquinas e Metais. São Paulo, Aranda Editora, $\mathrm{N}^{\circ}$ 401, p.76-89, 1999.

[3] GRAJEWSKI, Damian; GÓRSKI, Filip; ZAWADSKI, Przemyslaw; HAMROL, Adam. Application of virtual reality techniques in design of ergonomic manufacturing workplaces. Procedia Computer Science International Conference on Virtual and Augmented Reality in Education - Elsevier, Puerto de la Cruz, V.25, p. 289-301, 2013.

[4] TOLSTEDT, Jonathan. L. Prototyping as a means of requeriments elicitation. SAE International Off-Highway Congress, Las Vegas, N²002-01-1466, 2002.

[5] FERREIRA, Aurelio Buarque de Holanda. O dicionário Aurélio eletrônico - Século XXI. Versão 3.0. Rio de Janeiro, Lexikon Informática / Nova Fronteira, 1999. CD-ROM.

[6] SILVA, Guilherme Canuto da; KAMINSKI, Paulo Carlos. Selection of virtual and physical prototypes in the product development process. International Journal of Advanced Manufacturing Technology - Springer-Verlag, London, V.84, p. 1513-1530, 2016.

[7] KOTNIS, Mahesh A. Composite material for rapid tooling produced by high speed CNC machinery. Plastics: Bridging the Millennia, Proceedings of the SPE $57^{\text {th }}$ Annual Technical Conference \& Exhibits, Houston, p. 3327-3331, 1999.

[8] CAMBURN, Bradley Adam; ARLITT, Ryan; PEREZ, K. Blake; ANDERSON, David; CHOO, Pui Kun; LIM, Terry; GILMOUR, Adam; WOOD, Kristin. Design prototyping of systems. $21^{\text {st }}$ International Conference on Engineering Design, Vancouver, 2017.

[9] ZWAANENBURG, Koos. Integration of physical and virtual prototypes. SAE World Congress, Detroit, N²002-01-1290, 2002.

[10] CRRC Corporation Limited web site. Protótipo virtual do bogie. Disponível em http://www.crrcgc.cc/pt/g15853/m32120/t282727.aspx, 2017. Acesso em 25/05/2017.

[11] MACARRÃO JUNIOR, Leonardo; KAMINSKI, Paulo Carlos. Uma avaliação técnica e econômica do uso de mock-ups e de prototipagem rápida no processo de 
desenvolvimento do produto. $12^{\circ}$ Congresso e Exposição Internacionais de Tecnologia da Mobilidade, São Paulo, N²003-01-3640, 2003.

[12] Portal Ergonomia no Trabalho. O que é Ergonomia? Disponível em http://www.ergonomianotrabalho.com.br/ergonomia.html. Acesso em 15/05/2017.

[13] GUÉRIN, F.; LAVILlE, A.; DANIELLOU, F.; DURAFFOURG, J.; KERGUELEN, A. Tradução: INGRATTA, Giliane M. J.; MAFFEI, Marcos. Compreender o trabalho para transformá-lo. A prática da ergonomia. São Paulo. Editora Blucher, 2008. 201p.

[14] KRÜGER, Jörg; Automated vision-based live ergonomics analysis in assembly operations. CIRP Annals - Manufacturing Technology Journal - Elsevier, V.64, p. 9-12, 2015.

[15] FABBRO, Enrico del; SANTAROSSA, Daria. Ergonomic analysis in manufacturing process. A real time approach. $48^{\text {th }}$ CIRP Conference on Manufacturing Systems, Elsevier Procedia CIRP, Ischia, V.41, p. 957-962, 2016.

[16] ALTING, Leo. Manufacturing Engineering Processes. New York, Mercel Dekker Inc., 1994. 492p.

[17] BERRUECOS, Pablo. Ford reduce en 70\% su tasa de lesiones en la línea de producción. Dragster web site. Disponível em: http://dragstermx.com/www/2015/07/16/fordreduce-en-70-su-tasa-de-lesiones-en-la-linea-de-produccion/, 2015. Acesso em 27/05/2017.

[18] BARALDI, Emilio C.; KAMINSKI, Paulo C. Ergonomic planned supply in an automotive assembly line. Human Factors and Ergonomics in Manufacturing \& Service Industries Journal, V.21, 104-119, 2011.

[19] POLÁSEK, Patrik; BURES, Marek; SIMON, Michal. Comparison of Digital Tools for Ergonomics in Practice. $25^{\text {th }}$ DAAAM International Symposium on Intelligent Manufacturing and Automation - Elsevier Procedia Engineering, Zadar, V.100, 1277-1285, 2014.

[20] MOREIRA, Maria Edicy. Siemens PLM realiza simpósio sobre manufatura digital. Cadxpert web site. Disponível em: http://www.cadxpert.com.br/acontece/siemens-plmrealiza-simposio-sobre-manufatura-digital/, 2013. Acesso em 27/05/2017.

[21] MARZANO, A.; AGYAPONG-KODUA, K.; RATCHEV, S. Virtual Ergonomics and Time Optimization of a Railway Coach Assembly Line. $45^{\text {th }}$ Conference on Manufacturing Systems - Elsevier Procedia CIRP, V.3, p. 555-560, 2012.

[22] ZHOU, Dong; CHEN, Jiayu; LV, Chuan; CAO, Qingyuan. A method for integrating ergonomics analysis into maintainability design in a virtual environment. International Journal of Industrial Ergonomics - Elsevier, V.54, p. 154-163, 2016. 